\title{
Growth of aligned carbon nanotubes on carbon microfibers by dc plasma-enhanced chemical vapor deposition
}

\author{
L.-H. Chen, ${ }^{\text {a) }}$ J. F. AuBuchon, I.-C. Chen, C. Daraio, X.-R. Ye, A. Gapin, and S. Jin \\ University of California at San Diego, La Jolla, California 92093-0411 \\ C. M. Wang \\ Pacific Northwest National Laboratory, PO Box 999, Richland, Washington 99352
}

(Received 12 August 2005; accepted 22 November 2005; published online 18 January 2006)

\begin{abstract}
It is shown that unidirectionally aligned carbon nanotubes can be grown on electrically conductive network of carbon microfibers via control of buffer layer material and applied electric field during dc plasma chemical vapor deposition growth. Ni catalyst deposition on carbon microfiber produces relatively poorly aligned nanotubes with significantly varying diameters and lengths obtained. The insertion of $\mathrm{Ti} 5 \mathrm{~nm}$ thick underlayer between $\mathrm{Ni}$ catalyst layer and $\mathrm{C}$ microfiber substrate significantly alters the morphology of nanotubes, resulting in much better aligned, finer diameter, and longer array of nanotubes. This beneficial effect is attributed to the reduced reaction between $\mathrm{Ni}$ and carbon paper, as well as prevention of plasma etching of carbon paper by inserting a Ti buffer layer. Such a unidirectionally aligned nanotube structure on an open-pore conductive substrate structure may conveniently be utilized as a high-surface-area base electrodes for fuel cells, batteries, and other electrochemical and catalytic reactions. () 2006 American Institute of Physics.
\end{abstract}

[DOI: 10.1063/1.2166472]

Carbon nanotubes (CNTs) have emerged as a new and attractive class of materials with unique electrical, mechanical, and various physical and chemical properties. ${ }^{1-3}$ Potential applications include nanoelectronic devices, ${ }^{4-7}$ catalyst supports, ${ }^{8}$ storage materials for hydrogen and other gases, ${ }^{9,10}$ and probe tips for atomic force microscope (AFM). ${ }^{2,11}$ While there have been a very large number of publications on nanotube growth and their applications, engineering the shape of CNTs is an important issue for successful applications of nanotubes. Aligned and well separated CNT morphology is important for many potential applications, where a high electric-field concentration is needed as in field emission applications, and where a large surface area is desirable as in catalytic reactions, such as fuel cell or battery applications.

The growth of CNTs by dc plasma-enhanced chemical vapor deposition (CVD) involves many processing parameters, such as bias field, plasma power, temperature, chamber pressure, and feed gas composition. In general, the diameter and distribution of CNTs synthesized by CVD processing are dependent on the morphology of catalyst particles or layer. The shape of CNTs can be controlled by the applied bias voltage, the nature of catalyst and buffer layer deposition as well as the composition of plasma in a dc plasma-enhanced CVD process. ${ }^{12,13}$ The growth direction of the nanotubes can be controlled by the electrical field related to either applied bias or plasma induced bias, which is often perpendicular to the substrate surface. More recently, multiple sharp bending of CNTs to produce a zig-zag morphology has also been demonstrated by repeatedly altering the applied field directions. ${ }^{14}$ Such an alignment of CNTs has been demonstrated mostly on flat surfaces, and there have been few reports on aligned CNT growth on a fiber-configured substrate.

It is well known that CNT nucleation and growth occurs mostly on semiconductive Si substrate as well as electrically

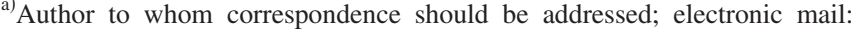
lhchen@ucsd.edu
}

insulating ceramic substrates. Reports of CNT growth on conductive substrates has been scarce up until now. The lack of high electrical conductivity of substrate limits the applications of CNTs in many applications including electrochemical reactions or electronic interconnection applications. If the CNT arrays can be grown on a conductive substrate, and in a well aligned and mutually separated configuration, it can be very useful for applications, such as fuel cells, whereas as a high-surface-area electrode support for catalyst particle loading. Carbon microfibers which constitute what is commonly known as "carbon paper" has highly porous and electrically conductive characteristics, which can be a good candidate for the ideal three-dimensional electrode support if nanotubes with high surface area can be grown with an optimum morphology. Recently, nonaligned CNT were grown on carbon papers by thermal CVD process for fuel cell applications. ${ }^{15}$ In the present work, we demonstrate the growth of vertically aligned and well separated CNTs on electrically conductive carbon microfiber by using the dc plasma-enhanced CVD technique, and discuss the effect of processing parameters on nanotube morphology.

Vertically aligned CNTs with different morphologies were grown by dc plasma-enhanced chemical vapor deposition process on a carbon paper substrate (procured from ElectroChem. Inc., Woburn, MA). A catalyst layer coating of either nickel only $(\sim 5-15 \mathrm{~nm}$ thick) or a double layer of titanium deposition followed by nickel deposition $(\mathrm{Ti} / \mathrm{Ni}$ $\sim 5-10 \mathrm{~nm} / 5-15 \mathrm{~nm}$ ) was added by sputter deposition on the carbon paper substrates. Prior to the onset of nanotube growth, the substrate with the catalyst film was heated to $\sim 700{ }^{\circ} \mathrm{C}$ in a $\mathrm{H}_{2}$ atmosphere to break up the $\mathrm{Ni}$ film into isolated nanoislands with an average diameters of $30-150 \mathrm{~nm}$ depending on the nature of the catalyst metal deposition. The base pressure of the CVD chamber was maintained at $\sim 5 \times 10^{-2}$ Torr during the $\mathrm{H}_{2}$ treatment. Ammonia gas $\left(\mathrm{NH}_{3}\right)$ was then fed into the chamber to replace the $\mathrm{H}_{2}$ gas. A dc bias of 450, 500, 550, or $600 \mathrm{~V}$ was applied 

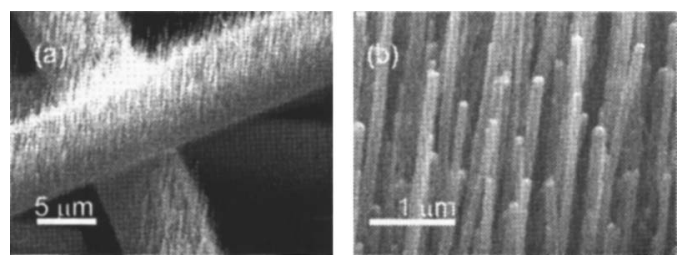

FIG. 1. SEM micrographs showing the overall configuration of the undirectionally aligned CNTs on carbon microfiber substrate: (a) Low magnification, (b) Higher magnification.

between the anode and the cathode to create the plasma and grow CNTs with different morphologies. The distance between anode and cathode was kept at $\sim 1 \mathrm{~cm}$. The acetylene gas $\left(\mathrm{C}_{2} \mathrm{H}_{2}\right)$ was then added to the chamber to start the growth of multiwall nanotubes, with a flow rate at $30 \mathrm{sccm}$ with the total $\mathrm{NH}_{3}$ and $\mathrm{C}_{2} \mathrm{H}_{2}$ pressure at $\sim 3$ Torr. After being held in the plasma for $\sim 5-20 \mathrm{~min}$, nanotube growth on the carbon microfibers with a typical length of $\sim 0.5-2 \mu \mathrm{m}$. Field emission scanning electron microscopy (SEM) was performed with FEI microscope to evaluate the microstructural morphology of the nanotubes grown. High-resolution transmission electron microscope (TEM) and energy dispersion spectrometry (EDX) were carried out with Jeol JEM 2010 microscope operated at $200 \mathrm{keV}$ to analyze the structure and composition of carbon nanostructure.

The carbon paper substrate is composed of carbon microfibers with an average diameter of $\sim 5 \mu \mathrm{m}$. A threedimensional network structure is formed with the carbon microfibers randomly arranged and bonded by a paste. To grow aligned CNTs on a carbon microfiber substrate, a $10 \mathrm{~nm}$ thick Ni film was sputter deposited as a catalyst layer. The CNT growth was carried out using an applied bias voltage from $450 \mathrm{~V}$ to $600 \mathrm{~V}$. All other processing parameters including the growth temperature, heating time, and ammonia/ acetylene gas composition were kept constant.

Shown in Figs. 1(a) and 1(b) are the SEM micrographs depicting the overall configuration of the undirectionally aligned CNTs on a conductive carbon microfiber substrate. The CNTs were grown with $10 \mathrm{~nm} \mathrm{Ni}$ on $5 \mathrm{~nm}$ Ti buffer layer at a CVD temperature of $700{ }^{\circ} \mathrm{C}$, and a dc bias voltage at $500 \mathrm{~V}$. It is evident from the figure that the CNTs nucleate and grow well on a carbon microfiber with a desirable configuration of unidirectional alignment toward the anode direction of the applied electric field, mutually separated from each other without any sign of agglomeration. Such a separation is important in order to deposit electrolytic catalyst nanoparticles (such as $\sim 5 \mathrm{~nm}$ size Pt nanoparticles) on the nanotube surface in high density, and also to ensure that all of the Pt nanoparticles are exposed for maximum catalytic reaction.

The morphology of CNTs grown on carbon microfiber depends much on processing details. Figures 2(a)-2(d) shows the SEM images of CNT samples for 20 min growth as a function of applied bias voltage. While some alignment of nanotubes occurs, the degree of alignment does not appear to be outstanding. The diameter and length of nanotubes show a significant variation. The striking difference in CNT morphology induced by the voltage change is apparent from the figures. At a lower bias field of $450 \mathrm{~V}$, nanotubes shorter than $1 \mu \mathrm{m}$ were grown vertically as shown in Fig. 2(a). The $\mathrm{Ni}$ catalyst particles with significantly varied diameters are present on top of all nanotubes indicating the occurrence of Downloaded 28 Sep 2006 to 131.215.225.9. Redistribution subject
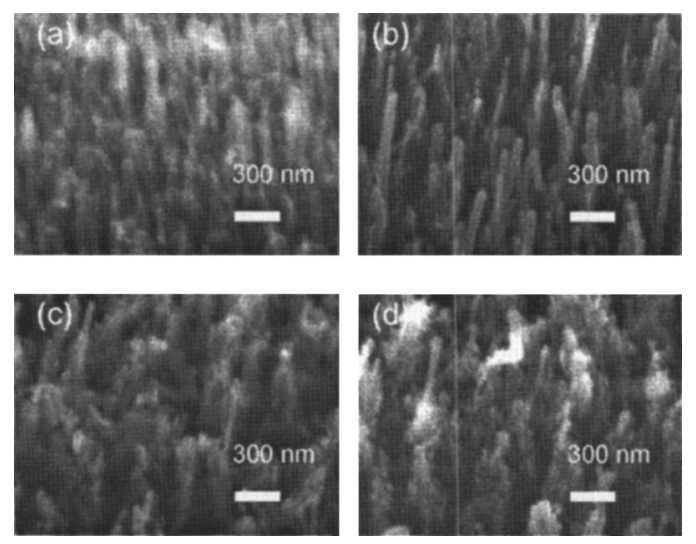

FIG. 2. SEM images of CNT samples grown on Ni $(10 \mathrm{~nm})$ coated carbon paper substrate at a dc bias of (a) $450 \mathrm{~V}$, (b) $500 \mathrm{~V}$, (c) $550 \mathrm{~V}$, and (d) $600 \mathrm{~V}$, respectively, for $20 \mathrm{~min}$.

tip-growth mechanism. In contrast to the $450 \mathrm{~V}$ sample, the $500 \mathrm{~V}$ sample [Fig. 2(b)] shows the longer and forestlike nanotube structure, with length more than $1.5 \mu \mathrm{m}$ and better alignment. It is also noticeable that there are still some short tubes grown on the substrate, which exhibit a large variation in diameter. For a higher applied voltage of 550 V, Fig. 2(c), short nanotubes with small diameter, ranging from 20 to $50 \mathrm{~nm}$, grow together and form a clumplike structure. In addition to the main nanotubes, some ultrafine nanotubes with a diameter of less than $10 \mathrm{~nm}$, are observed. At an even higher bias of $600 \mathrm{~V}$, Fig. 2(d), nanotube clumps grow longer into a Christmas tree shape, in which more ultrafine branched nanotubes grow together with coarse nanotubes.

To modify the morphology of CNTs grown on carbon microfibers, we add a Ti buffer layer of $50 \mathrm{~A}$ under the 100 A Ni catalyst layer. Figures 3(a)-3(d) show the SEM images of CNT samples. At lower bias fields of $450 \mathrm{~V}$ and $500 \mathrm{~V}$, similar aligned and nonuniformly distributed nanotubes were grown from the carbon paper substrate. However, the CNT diameter gets smaller and more uniform, with their length increasing noticeably As a result, the nanotube distribution density also becomes higher compared to that for CNTs grown from the pure Ni catalyst. For a higher applied voltage of $550 \mathrm{~V}$, Fig. 3(c) long and well-aligned nanotubes with uniform and small diameter, ranging from 30 to $50 \mathrm{~nm}$, are obtained, which is different from the clumplike structure of nanotubes grown from Ni catalyst. Around main nano-
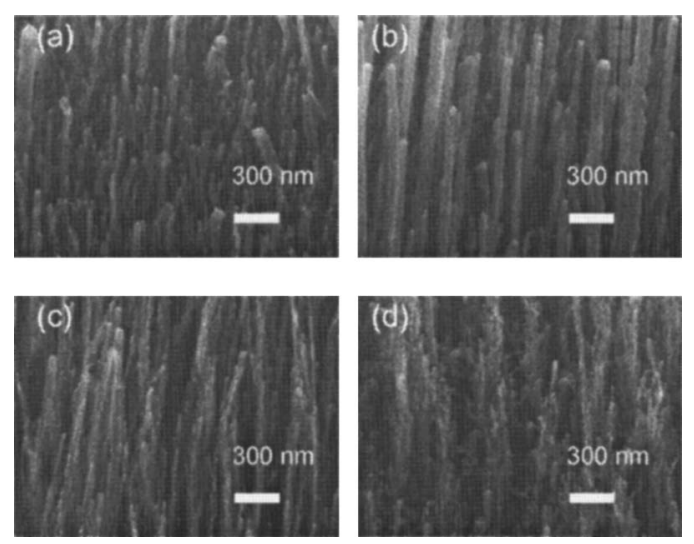

FIG. 3. SEM images of CNT samples grown on $\mathrm{Ti}(5 \mathrm{~nm}) / \mathrm{Ni}(10 \mathrm{~nm})$ coated carbon paper substrate at a dc bias of (a) $450 \mathrm{~V}$, (b) $500 \mathrm{~V}$, (c) $550 \mathrm{~V}$, and (d) $600 \mathrm{~V}$, respectively, for $20 \mathrm{~min}$.

o AIP license or copyright, see http://apl.aip.org/apl/copyright.jsp 

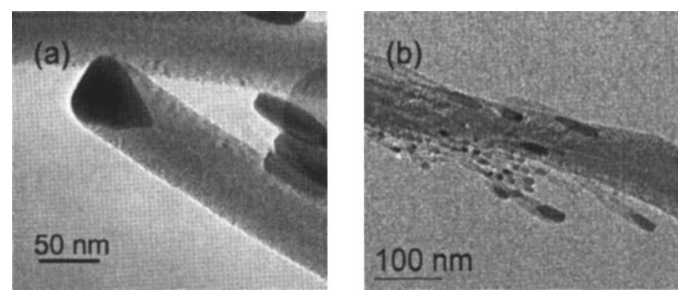

FIG. 4. TEM images of CNTs, grown at (a) $500 \mathrm{~V}$ and (b) $550 \mathrm{~V}$, respectively, for $20 \mathrm{~min}$.

tubes, some ultrafine nanotubes are occasionally observed. At an even higher bias of $600 \mathrm{~V}$, Fig. 3(d), the growth of ultrafine nanotubes is more significant and they form a clumplike nanotube structure again, although they are still taller than those grown from pure Ni catalysts. As seen in Figs. 2 and 3, a significant improvement of nanotube growth morphology, such as diameter, length, and distribution, is obtained by addition of Ti buffer layer. The average diameter of the catalyst particles from the $\mathrm{Ni}$ film ranges from $30-100 \mathrm{~nm}$, which is decreased by the addition of Ti layer. The density and size distribution of catalyst particles improves with the addition of Ti. Such effects are ascribed to the change of adhesion of metal on carbon fiber as well as the prevention of any undesirable reaction between $\mathrm{Ni}$ and carbon microfibers by the insertion of a Ti layer between carbon fiber and $\mathrm{Ni}$ catalyst layer. It is also worth noting that the growth rate of nanotubes is raised by the insertion of a Ti layer under the same processing conditions. The nanotube growth rate is determined by the number of carboncontaining species impinging on the catalyst surface, by the rate of their adsorption, decomposition, and desorption of reaction byproducts, and by the diffusion and precipitation of carbon species. As reported in previous papers, there must be an optimum plasma composition for a high growth rate. ${ }^{16,17}$ For the growth of nanotubes directly on carbon microfibers, the occurrence of plasma etching in plasma-enhanced CVD growth of nanotubes on these carbon microfibers creates the additional carbon-contained species, which modifies the composition of the resultant species and consequently changes the growth rate.

TEM images of the samples grown from $\mathrm{Ti} / \mathrm{Ni}$ catalyst at $500 \mathrm{~V}$ and $550 \mathrm{~V}$, respectively, for $20 \mathrm{~min}$, are shown in Figs. 4(a) and 4(b). Figure 4(a) shows the nanotubelike structure with a Ni catalyst cap on top of carbon nanotubes. There is no obvious amorphous carbon layer observed which covers the Ni catalyst. This implies that longer tubes can be obtained if a longer CVD growth time is given. EDX spot analysis indicates that the nanotube matrix contains mostly carbon and trace of Ni. We also learn that catalyst particles are composed of mostly $\mathrm{Ni}$, where no detectable $\mathrm{Ti}$ is found. Fig. 4(b) is the TEM micrograph of a main nanotube of $\sim 100 \mathrm{~nm}$ in diameter from the $550 \mathrm{~V}$ sample, where a few tiny nanotubes of $\sim 5-10 \mathrm{~nm}$ branch are also formed. The main nanotube is crystalline and mostly formed by carbon. The tiny inclusions, which may be present either inside or on the surface of main nanotube, were identified to be mainly composed of Ni. It is most likely that the small Ni particles, as well as the growth of secondary small nanotubes, are caused by sputtering (and redeposition) of the main catalyst particles during higher voltage bias CVD. Further microstructural analysis is required to fully understand the detailed structure of the branched nanotubes.

In summary, the growth of vertically aligned and well separated CNT array on an electrically conductive carbon microfiber substrate has been demonstrated by using the dc plasma-enhanced CVD technique. Desirable CNT morphology can be engineered by insertion of an appropriate buffer layer and control of applied voltage during dc plasma CVD growth to cause field-induced alignment, yet avoid the formation of secondary nucleation of finer nanotubes and associated clumping. Such a unidirectionally aligned nanotube structure on an open-pore conductive substrate structure may conveniently be utilized as a three-dimensionally configured, high-surface-area base electrodes for fuel cells, batteries, and other electrochemical and catalytic reactions.

The authors acknowledge the support of the work by NSF-NIRTs under Grant Nos. DMI-0210559 and DMI0303790, and University of California Discovery Fund under Grant No. ele02-10133/Jin.

${ }^{1}$ S. Iijima, Nature (London) 354, 56 (1991).

${ }^{2}$ H. Dai, J. H. Hafner, A. G. Rinzler, D. T. Colbert, and R. Smalley, Nature (London) 384, 187 (1996).

${ }^{3}$ L. Forro and C. Schnonenberger, Carbon Nanotubes Synthesis, Structure, Properties and Applications (Springer, New York, 2001), p. 329.

${ }^{4}$ T. Hertel, R. Martel, and P. Avouris, J. Phys. Chem. B 102, 910 (1998).

${ }^{5}$ M. A. Guillorn, A. V. Melechko, V. I. Merkulov, E. D. Ellis, M. L. Simpson, L. R. Baylor, and G. J. Bordonaro, J. Vac. Sci. Technol. B 19, 2598 (2001).

${ }^{6}$ C. Bower, W. Zhu, D. Shalom, D. Lopez, L. H. Chen, P. L. Gammel, and S. Jin, Appl. Phys. Lett. 80, 3820 (2002).

${ }^{7}$ K. B. Teo, M. Chhowalla, G. A. Amaratunga, W. I. Miline, P. Legagneux, G. Pirio, L. Gangloff, D. Pribat, V. Semet, V. T. Binh, W. H. Bruenger, J. Eichholz, H. Hanssen, D. Friedrich, S. B. lee, D. G. Hasko, and H. Ahmed, J. Vac. Sci. Technol. B 21, 693 (2003).

${ }^{8}$ J. M. Planeiz, N. Coustel, B. Coq, V. Brotons, P. S. Kumbhar, R. Dutartre, P. Geneste, P. Bernier, and P. M. Ajayan, J. Am. Chem. Soc. 116, 7935 (1994).

${ }^{9}$ A. C. Dillon, K. M. Jones, T. A. Bekkedahl, C. H. Kiang, D. S. Bethune, and M. J. Heben, Nature (London) 386, 377 (1997).

${ }^{10}$ G. E. Gadd, M. Blackfold, S. Moricca, N. Webb, P. J. Evans, A. M. Smith, G. Jacobsen, S. Leung, A. Day, and Q. Hua, Science 277, 933 (1997).

${ }^{11}$ S. S. Wong, J. D. Harper, P. T. Lansbury, and C. M. Lieber, J. Am. Chem. Soc. 120, 603 (1998).

${ }^{12}$ L.-H. Chen, J. F. AuBuchon, A. Gapin, C. Daraio, P. Bandaru, S. Jin, D. W. Kim, and I. K. Yoo, Appl. Phys. Lett. 85, 5373 (2004).

${ }^{13}$ M. Chhowalla, K. B. K. Teo, C. Ducati, N. L. Rupesinghe, G. A. J. Amaratunga, A. C. Ferrari, D. Roy, J. Robertson, and W. I. Milne, J. Appl. Phys. 90, 5308 (2001).

${ }^{14}$ J. F. AuBuchon, L.-H. Chen, A. I. Gapin, D.-W. Kim, C. Daraio, and S. Jin, Nano Lett. 4, 1781 (2004).

${ }^{15}$ C. Wang, M. Waje, X. Wang, J. M. Tang, R. C. Haddon, and Y. Yan, Nano Lett. 4, 345 (2004).

${ }^{16}$ N. R. Franklin, Y. Li, R. J. Chen, A. Javey, and H. Dai, Appl. Phys. Lett. 79, 4571 (2001)

${ }^{17}$ D. B. Hash and M. Meyyappan, J. Appl. Phys. 93, 750 (2003). 